\title{
Liver dysfunction as predictor of prognosis in patients with amyloidosis: utility of the Model for End-stage Liver disease (MELD) scoring system
}

\author{
Francesco Cappelli $^{1,3}$ - Samuele Baldasseroni ${ }^{1,2}$ - Franco Bergesio ${ }^{3}$ - Valentina Spini ${ }^{1}$. \\ Alessia Fabbri ${ }^{5} \cdot$ Paola Angelotti $^{5} \cdot$ Elisa Grifoni $^{4} \cdot$ Paola Attanà $^{4} \cdot$ Francesca Tarantini $^{2}$ • \\ Niccolò Marchionni ${ }^{2} \cdot$ Alberto Moggi Pignone $^{4} \cdot$ Federico Perfetto $^{3,4}$
}

Received: 18 March 2016/Accepted: 1 July 2016/Published online: 1 August 2016

(C) SIMI 2016

\begin{abstract}
Amyloidosis prognosis is often related to the onset of heart failure and a worsening that is concomitant with kidney-liver dysfunction; thus the Model for Endstage Liver disease (MELD) may be an ideal instrument to summarize renal-liver function. Our aim has been to test the MELD score as a prognostic tool in amyloidosis. We evaluated 128 patients, 46 with TTR-related amyloidosis and 82 with AL amyloidosis. All patients had a complete clinical and echocardiography evaluation; overall biohumoral assessment included troponin I, NT-proBNP, creatinine, total bilirubin and INR ratio. The study population was dichotomized at the 12 cut-off level of MELD scores; those with MELD score $>12$ had a lower survival
\end{abstract}

F. Cappelli and S. Baldasseroni equally contributed to the present manuscript.

Electronic supplementary material The online version of this article (doi:10.1007/s11739-016-1500-0) contains supplementary material, which is available to authorized users.

Francesco Cappelli

cappellifrancesco@inwind.it

1 Intensive Cardiac Unit, Department of Heart and Vessels, University of Florence and Azienda Ospedaliero-

Universitaria Careggi [AOUC], Largo Brambilla 3, 50134 Florence, Italy

2 Research Unit of Medicine of Aging, Department of Experimental and Clinical Medicine, University of Florence, Florence, Italy

3 Regional Amyloid Centre, Azienda OspedalieroUniversitaria Careggi, Florence, Italy

4 Department of Internal Medicine, University of Florence, Florence, Italy

5 Department of Heart and Vessels, University of Florence, Florence, Italy compared to controls in the study cohort (40.7 vs $66.3 \%$; $p=0.006$ ). Either as a continuous and dichotomized variable, MELD shows its independent prognostic value at multivariable analysis $(\mathrm{HR}=1.199, \quad 95 \% \quad \mathrm{CI}$ 1.082-1.329; HR $=2.707,95 \%$ CI 1.075-6.817, respectively). MELD shows a lower prognostic sensitivity/ specificity ratio than troponin I and NT-proBNP in the whole study population and AL subgroup, while in TTR patients MELD has a higher sensitivity/specificity ratio compared to troponin and NT-proBNP (ROC analysisAUC: 0.853 vs 0.726 vs 0.659 ). MELD is able to predict prognosis in amyloidosis. A MELD score $>12$ selects a subgroup of patients with a higher risk of death. The predictive accuracy seems to be more evident in TTR patients in whom currently no effective scoring systems have been validated.

Keywords Amyloid · Prognosis · MELD · Liver dysfunction

\section{Introduction}

Different risk scores, biohumoral and clinical variables have been evaluated as predictors of prognosis in patients affected by amyloidosis [1-4]. The majority of them are focused on the cardiovascular system, such as atrial peptides [5, 6], troponin [6, 7] and echocardiographic indexes of left and right ventricular function [8-12]. They independently predict the prognosis of this systemic infiltrative multi-organ disease.

The progression and worsening of amyloidosis is due to the onset of heart failure [13], often associated with kidney or liver dysfunction; thus the composite Model for Endstage Liver disease (MELD) [14], based on patient's level 
of creatinine, total bilirubin and International Normalized Ratio-INR, may be an useful instrument to summarize the global renal and liver function.

The MELD scoring system usually adopted for prioritizing cirrhotic patients candidates for liver transplantation [15] has been recently demonstrated to predict the prognosis in outpatients with heart failure [16], in those candidates for ventricular assistance devices [17, 18], or to tricuspid valve surgery or orthotopic heart transplantation [19-21].

In amyloidosis patients, the kidney and liver dysfunction may be caused by different mechanisms, in part linked to the direct infiltrative deposition of amyloid mostly in AL forms, and the rest to the onset of cardio-hepatic [22] or cardio-renal syndromes [23] associated with the presence of heart failure. The aforementioned syndromes are usually characterized by the contemporary presence of venous congestion and arterial hypoperfusion; both the hemodynamic alterations are related to the presence of cardiac biventricular dysfunction [23].

In this context, we have already demonstrated the prognostic role of right ventricular dysfunction in patients with AL amyloidosis [12, 24]; however, the pathophysiological pathway is not fully understood. There may be a possible link in the progressive worsening of renal and liver function.

On this basis our aims have been to demonstrate, first, the predictability of the MELD score in amyloidosis patients, and second to test if its prognostic value is influenced by the etiology of the amyloidosis.

\section{Methods}

\section{Data collection}

From the database of Regional Center of Amyloidosis of the University of Florence, Italy, between January 2006 and June 2013, we retrospectively evaluated 154 patients referred to our amyloidosis referral center for clinical and instrumental evaluation. One patient was excluded because he was already on dialysis; five excluded patients were on anticoagulant therapy with warfarin at the entry visit due to atrial fibrillation; moreover in 16 patients one or more of the MELD variables were unavailable at diagnosis; and in four patients biohumoral variable analysis exceeded the 30-day temporal window from echocardiography evaluation. The study population was therefore composed of 128 patients, 46 with TTR-related amyloidosis (28 with ATTR wild type and 18 with ATTR mutated) and 82 with AL amyloidosis. Diagnosis of AL amyloidosis was made by biopsy of an involved organ, which demonstrated the typical Congo red birefringence when viewed under polarized light. The positive biopsy site was abdominal fat in 53 patients $(65 \%)$, kidney in $12(14 \%)$, myocardium in 12 $(14 \%)$, and salivary gland in $5(7 \%)$. AL amyloidosis was confirmed by the finding of a monoclonal protein in the serum or urine or a monoclonal population of plasma cells in the bone marrow, when evaluated by immunohistochemistry in the absence of any TTR mutation at DNA analysis. In two cases with solitary myocardial involvement, electron microscopy with immunogold labeling was used to unambiguously characterize amyloid fibrils.

Diagnosis of ATTR mutated was based on genotyping [25], and tissue biopsy of abdominal fat in 10 patients $(56 \%)$, myocardium in $5(28 \%)$, and salivary gland in 3 $(16 \%)$. ATTR mutations were: Ile68Leu $(n=11$ patients, $61 \%)$, Val122Ile ( $n=5$ patients $28 \%)$, Glu54Val $(n=1$ patients), Gly57Arg ( $n=1$ patients). All 18 of the patients showed the characteristic increased myocardial uptake of 99mTc-3,3-diphosphono-1,2-propanodicarboxylic (DPD) acid scintigraphy, further confirming the diagnosis of TTRrelated cardiac amyloidosis [26].

Diagnosis of ATTR wild type was made by tissue biopsy or positive DPD scintigraphy in absence of TTR mutation and of a plasma cell dyscrasia. A total of 19 patients $(68 \%)$ had histological proof of ATTR amyloidosis by Congo red and immunohistochemical staining of myocardial $(n=6,31 \%)$ or other tissues $(n=13 ; 69 \%$; abdominal fat in 6 patients, carpal tunnel biopsy in 4 , and salivary gland in 3). In 9 patients (32\%) with negative tissue biopsy, definite cardiac ATTR wild type was defined as intense $99 \mathrm{mTc}$-DPD uptake in heart (grade 2 or 3 as defined by Perugini et al.) [26] in the absence of a plasma cell dyscrasia and TTR/AApoAI mutation; in the latter patients a cardiac MRI was performed with a late gadolinium enhancement (LGE) consistent with cardiac amyloid involvement.

All patients gave written informed consent for their clinical records to be used for research purposes, in accordance with Institutional Review Board guidelines.

NT-proBNP was measured with an electrochemiluminescence sandwich immunoassay (ECLIA, Roche) in the central hospital laboratory. Troponin I measurements were performed by immunochemiluminescence assay using a Centaur XP (Siemens Healthcare, Erlangen, Germany). Creatinine, total bilirubin and International Normalized Ratio were collected from central hospital laboratory. Both systolic and diastolic arterial blood pressure were evaluated at the entry visit in the Registry.

\section{Standard and TDI echocardiography}

In a temporal window of 30 days from biohumoral analysis, patients were referred to our laboratory for M-mode, 2-dimensional, conventional and tissue Doppler 
echocardiographic study. Echocardiography was performed by a single experienced operator (FC), blinded to the clinical history of the patient, using a Vivid 7 System (Vingmed, General Electric, Horten, Norway) equipped with a $3 \mathrm{~S}$ probe. At least three consecutive beats were recorded, and the images were digitized and analyzed offline. According to the standards of the American Society of Echocardiography [27] the following parameters were assessed: end-diastolic thickness of ventricular septum (IVS) and LV posterior wall (PW), LV end-diastolic and end-systolic diameters (LV EDD and LV ESD, respectively), body surface area (BSA)-indexed LV mass (LVmass $_{\text {ind }}$ ), LV endocardial fractional shortening (FS), left atrial area (LAA, evaluated from the apical four chamber view at the end of systole), LV end-diastolic and endsystolic volumes (LV EDV and LV ESV, respectively), ejection fraction (EF, estimated with the biplane Simpson method), mitral peak flow velocity in early and late diastole (E and A, respectively, during atrial contraction), E wave deceleration time (DT), E/A ratio, RV free wall thickness (RV FW), RV end-diastolic diameter (RV EDD, evaluated from parasternal long axis view) and the systolic displacement of the lateral portion of the tricuspid annular plane (TAPSE).

Pulmonary artery systolic pressure (PASP) was approximated by adding to trans-tricuspid pressure gradient an estimate of right atrial pressure assessed by inferior vena cava dimension and respiratory variation. We also evaluated pulsed TDI-derived early diastolic peak velocity at lateral mitral annulus (E'), as an index of LV relaxation, and $\mathrm{E} / \mathrm{E}$ ' ratio, as an index of $\mathrm{LV}$ filling pressure.

\section{MELD scoring system}

The standard MELD score was defined according to the following equation: $11.2 \times(\operatorname{Ln} I N R)+0.378 \times(\operatorname{Ln}$ total bilirubin $)+0.957 \times($ Ln creatinine $)+0.643[14]$.

\section{Statistical analysis}

Our analyses were performed by using the $\operatorname{SPSS}^{\circledR}$ for Windows package version 19 (SPSS Inc., Chicago, IL, USA). Categorical and continuous variables were expressed as frequencies (percentages) and as mean \pm standard deviation, respectively. Categorical comparisons were used for comparison between groups. The cut-off level of MELD was pointed out at 12 according to Kim MS and colleagues data [16].

The sensitivity and specificity of this predefined cutoff in all-cause death prediction was tested using area under the receiver operating characteristics curve (ROC) analysis in whole study population and subgroups of patient according to amyloid etiology. We calculated the area under the curve of MELD comparing it with AUC of NTproBNP that we know to be the strongest predictor of prognosis in amyloidosis [13].

The survival rate of the two MELD scoring groups was compared by using Kaplan-Meier curves with a log-rank test in the whole population and subgroups of patient according to amyloid etiology.

Two different models of Cox proportional hazard analysis were used to verify the association of MELD cutoff and MELD as an ordinal variable. In the multivariable model we introduced those variables associated with outcome in univariate analysis and those we considered relevant according to literature [13]. Among variables that had a predictive value on mortality at univariate analysis, but with similar clinical significance, only one was introduced in the multivariate logistic regression model, to avoid colinearity.

For all analyses the $\mathrm{p}$ value was pointed out at $<0.05$, the INPUT was 0.05 and the OUTPUT was 0.10 for all multivariable Cox regression models.

\section{Results}

Our study population was composed of 128 patients including 82 patients with systemic AL amyloidosis and 46 patients with TTR-related amyloidosis (28 with ATTR wild type and 18 with ATTR mutated).

The mean age of the study population was 71 years, 78 $(60 \%)$ were males, mean ejection fraction was $55.1 \pm 11.1 \%, 46(35.9 \%)$ were in advanced (III-IV) NYHA class, the mean level of creatinine, bilirubin and INR were $1.18 \pm 0.50 \mathrm{mg} / \mathrm{dl}, \quad 0.82 \pm 0.50 \mathrm{mg} / \mathrm{dl}$, $1.14 \pm 1.03$, respectively. The mean MELD score was $10.1 \pm 3.7$. Our population presented significant diastolic dysfunction assessed by E' and E/E' $5.3 \pm 1.9 \mathrm{~cm} / \mathrm{sec}$ and $16.4 \pm 7.3$, respectively. The indirect index of right ventricular function TAPSE was $18.4 \pm 4.6$.

Mean follow-up period was $22.2 \pm 20.0$ months; during this follow-up period we registered 50 deaths (39\%), one patient with ATTR mutated etiology died from a cerebral neoplasm, one patient with AL amyloidosis died from endstage renal failure, all other patients died from a cardiovascular reason (end-stage heart failure or sudden death). Thus the mortality outcome can be defined substantially as cardiovascular mortality.

\section{Univariate analysis}

The study population was dichotomized according to the 12 cut-off level of MELD and 28 patients (20\%) were in the group above the cut-off level (Table 1). Comparisons between groups revealed that patients with a higher MELD 
Table 1 Demographic, clinical, biohumoral and echocardiographic characteristics of the study population and in the two etiologies of amyloidosis according to MELD cutoff

\begin{tabular}{|c|c|c|c|c|c|c|c|c|c|}
\hline & \multicolumn{3}{|c|}{ All patients $(n=128)$} & \multicolumn{3}{|c|}{ AL patients $(N=82)$} & \multicolumn{3}{|l|}{$\begin{array}{l}\text { TTR patients } \\
(n=46)\end{array}$} \\
\hline & $\begin{array}{l}\text { MELD } \leq 12 \\
N=100\end{array}$ & $\begin{array}{l}\text { MELD > } 12 \\
N=28\end{array}$ & $p$ & $\begin{array}{l}\text { MELD } \leq 12 \\
N=69\end{array}$ & $\begin{array}{l}\text { MELD }>12 \\
N=13\end{array}$ & $p$ & $\begin{array}{l}\text { MELD } \leq 12 \\
N=31\end{array}$ & $\begin{array}{l}\text { MELD > } 12 \\
N=15\end{array}$ & $p$ \\
\hline Gender (m/f) & $61 / 39$ & $17 / 11$ & 0.900 & $35 / 34$ & $5 / 8$ & 0.470 & $26 / 5$ & $12 / 3$ & 0.740 \\
\hline Age years & $69.7 \pm 10.8$ & $75.3 \pm 8.5$ & 0.013 & $68.2 \pm 10.3$ & $71.3 \pm 7.7$ & 0.483 & $73.3 \pm 11.4$ & $78.7 \pm 6.1$ & 0.060 \\
\hline $\begin{array}{l}\text { Creatinine mg/ } \\
\text { dl }\end{array}$ & $1.01 \pm 0.30$ & $1.80 \pm 0.59$ & $<0.001$ & $1.04 \pm 0.34$ & $2.1 \pm 0.71$ & $<0.001$ & $0.95 \pm 0.2$ & $1.52 \pm 0.33$ & $<0.001$ \\
\hline Bilirubin mg/dl & $0.73 \pm 0.40$ & $1.10 \pm 0.67$ & 0.003 & $0.61 \pm 0.24$ & $1.01 \pm 0.83$ & 0.002 & $1.00 \pm 0.53$ & $1.31 \pm$ & 0.077 \\
\hline NR & $1.08 \pm 0.14$ & $1.39 \pm 0.44$ & 0.001 & $1.04 \pm 0.13$ & $1.16 \pm 0.20$ & 0.014 & $1.14 \pm 0.14$ & $1.59 \pm$ & 0.005 \\
\hline Nt-proBNP ng/l & $4252 \pm 6301$ & $9488 \pm 1147$ & 0.023 & $4637 \pm 4637$ & $11426 \pm 14951$ & 0.002 & $3364 \pm 4874$ & $6524 \pm 4381$ & 0.040 \\
\hline Troponin ng/l & $0.16 \pm 0.31$ & $0.20 \pm 0.21$ & 0.549 & $0.16 \pm 0.34$ & $0.19 \pm 0.30$ & 0.545 & $0.17 \pm 0.18$ & $0.18 \pm 0.10$ & 0.852 \\
\hline SBP mm Hg & $119.1 \pm 17.9$ & $126.5 \pm 13.1$ & 0.305 & $114.7 \pm 17.3$ & $108.6 \pm 16.5$ & 0.753 & $122 \pm 18$ & $126 \pm 13$ & 0.589 \\
\hline DBP $\mathrm{mm} \mathrm{Hg}$ & $70 \pm 10.8$ & $74.3 \pm 8.2$ & 0.316 & $70.6 \pm 11.7$ & $69.5 \pm 11.3$ & 0.872 & $69 \pm 10$ & $74 \pm 8$ & 0.298 \\
\hline BSA $m^{2}$ & $1.74 \pm 0.15$ & $1.74 \pm 0.14$ & 0.940 & $1.7 \pm 0.14$ & $1.69 \pm 0.16$ & 0.543 & $1.79 \pm 0.16$ & $1.77 \pm 0.11$ & 0.793 \\
\hline LVDD mm & $45.6 \pm 5.9$ & $45.3 \pm 4.8$ & 0.770 & $45.3 \pm 5.7$ & $43.8 \pm 5.8$ & 0.404 & $46.3 \pm 6.4$ & $46.4 \pm 3.6$ & 0.962 \\
\hline LVSD mm & $29.7 \pm 6.4$ & $31.4 \pm 6.4$ & 0.239 & $28.1 \pm 5.5$ & $28.2 \pm 5.5$ & 0.945 & $33.2 \pm 6.7$ & $34.1 \pm 5.9$ & 0.690 \\
\hline FS \% & $34.8 \pm 9.5$ & $30.7 \pm 10.6$ & 0.060 & $37.9 \pm 8.5$ & $35.7 \pm 10.5$ & 0.456 & $27.9 \pm 7.9$ & $26.7 \pm 9.1$ & 0.673 \\
\hline IVS mm & $14.5 \pm 3.6$ & $15.9 \pm 3.3$ & 0.067 & $13.2 \pm 3.2$ & $14.5 \pm 3.6$ & 0.216 & $17.6 \pm 2.6$ & $17.1 \pm 2.6$ & 0.543 \\
\hline LVPW mm & $13.9 \pm 3.0$ & $15.6 \pm 2.9$ & 0.009 & $12.9 \pm 2.8$ & $14.5 \pm 3.9$ & 0.092 & $16.0 \pm 2.1$ & $16.4 \pm 1.6$ & 0.530 \\
\hline LVmass I $\mathrm{g} / \mathrm{m}^{2}$ & $153.1 \pm 52.9$ & $178.1 \pm 51.1$ & 0.032 & $135.2 \pm 44.9$ & $151.0 \pm 43.0$ & 0.261 & $195.5 \pm 46.3$ & $199.7 \pm 47.6$ & 0.953 \\
\hline $\mathrm{LA}$ area $\mathrm{cm}^{2}$ & $22.6 \pm 5.5$ & $25.6 \pm 5.1$ & 0.014 & $21.2 \pm 4.9$ & $22.9 \pm 4.5$ & 0.270 & $26.0 \pm 5.3$ & $28.0 \pm 4.3$ & 0.242 \\
\hline LVEDV ml & $83.9 \pm 25.2$ & $84.7 \pm 17.8$ & 0.891 & $82.6 \pm 24.4$ & $77.4 \pm 17.1$ & 0.484 & $87.0 \pm 27.2$ & $90.5 \pm 16.6$ & 0.653 \\
\hline LVESV ml & $37.2 \pm 17.4$ & $42.9 \pm 17.8$ & 0.143 & $34.2 \pm 14.4$ & $33.5 \pm 11.0$ & 0.873 & $44.0 \pm 21.6$ & $50.5 \pm 20.2$ & 0.343 \\
\hline EF \% & $56.3 \pm 10.0$ & $50.7 \pm 13.7$ & 0.020 & $58.6 \pm 8.4$ & $56.9 \pm 10.8$ & 0.549 & $51.1 \pm 11.5$ & $45.7 \pm 14.1$ & 0.177 \\
\hline $\mathrm{E} / \mathrm{A}$ & $1.56 \pm 1.03$ & $1.70 \pm 0.82$ & 0.566 & $1.35 \pm 0.89$ & $1.32 \pm 0.70$ & 0.921 & $2.2 \pm 1.1$ & $2.2 \pm 0.67$ & 0.785 \\
\hline DT ms & $200 \pm 73$ & $176 \pm 74$ & 0.152 & $209 \pm 69$ & $198 \pm 107$ & 0.674 & $181 \pm 79$ & $159 \pm 26$ & 0.320 \\
\hline $\mathrm{E}^{\prime} \mathrm{cm} / \mathrm{s}$ & $5.5 \pm 2.0$ & $4.6 \pm 1.4$ & 0.055 & $5.8 \pm 2.1$ & $5.1 \pm 1.6$ & 0.321 & $4.8 \pm 1.7$ & $4.2 \pm 1.0$ & 0.232 \\
\hline $\mathrm{E} / \mathrm{e}^{\prime}$ & $15.6 \pm 7.1$ & $19.4 \pm 7.1$ & 0.020 & $14.6 \pm 7.6$ & $16.2 \pm 5.5$ & 0.493 & $18.1 \pm 5.3$ & $22.2 \pm 7.4$ & 0.047 \\
\hline RVFW mm & $7.4 \pm 1.7$ & $7.8 \pm 1.7$ & 0.254 & $6.8 \pm 1.5$ & $7.1 \pm 1.9$ & 0.692 & $8.5 \pm 1.7$ & $8.4 \pm 1.2$ & 0.894 \\
\hline RVEDD mm & $29.1 \pm 5.4$ & $29.7 \pm 6.5$ & 0.622 & $28.7 \pm 4.9$ & $25.3 \pm 4.5$ & 0.034 & $30.0 \pm 6.2$ & $33.5 \pm 5.7$ & 0.088 \\
\hline TAPSE mm & $18.9 \pm 4.3$ & $16.7 \pm 5.5$ & 0.030 & $19.6 \pm 4.3$ & $19.2 \pm 6.8$ & 0.772 & $17.1 \pm 3.5$ & $14.6 \pm 3.0$ & 0.031 \\
\hline PASP $\mathrm{mmHg}$ & $34.3 \pm 11.2$ & $39.7 \pm 11.6$ & 0.037 & $33.2 \pm 11.8$ & $35.3 \pm 10.2$ & 0.581 & $37.0 \pm 9.2$ & $42.9 \pm 11.9$ & 0.097 \\
\hline
\end{tabular}

$A$ late diastolic mitral peak flow velocity, $E$ early diastolic mitral peak flow velocity, $e^{\prime}$ early diastolic peak velocity at lateral mitral annulus, $F S$ fractional shortening, $E F$ ejection fraction, $I V S$ interventricular septum thickness, $L A$ left atrium, $L V$ left ventricular, $L V E D D$ LV end-diastolic diameter, $L V E D V$ LV end-diastolic volume, $L V E S D$ LV end-systolic diameter, $L V E S V$ LV end-systolic volume, $L V P W$ posterior wall thickness, $P A S P$ pulmonary artery systolic pressure, $R V$ right ventricular, RVEDD RV end-diastolic diameter, $R V F W$ RV free wall thickness, TAPSE tricuspid annulus systolic plane excursion

show increased LV mass and LA area, lower LV EF and worse LV diastolic function. Moreover, they demonstrate RV longitudinal dysfunction with an increase in pulmonary artery systolic pressure. On the other hand, no significant difference is observed in RV dimension and wall thickness. Patients with MELD $>12$ are significantly older with increased NT-proBNP plasma level while no significant differences are observed in troponin values. Gender distribution and arterial blood pressure values are superimposable between the two groups. The prevalence of a history of diabetes mellitus, arterial hypertension, coronary artery disease and peripheral artery disease does not differ between the groups. The baseline characteristics of our cohort are further categorized according to amyloidosis etiology (Table 1).

We observe fewer baseline differences between the dichotomized group in AL amyloidosis patients. In this subgroup, patients with a MELD score $>12$ present an increase in NT-proBNP plasma levels and increased RVEDD while no differences are observed in LV dimensions and function, TAPSE or PASP. On the other hand, in the TTR population, subjects with a MELD $>12$ show 
increased LV filling pressure assessed by E/e' ratio with increase PASP and reduce RV function.

\section{Prognostic findings}

As reported in Table 2, deceased patients show increased MELD $(11.5 \pm 4.6$ vs $9.1 \pm 2.7, p<0.001)$ and present a worse clinical, biohumoral and echocardiographic profile (Table 2). Results of the unadjusted Kaplan-Meier analysis comparing survival of patients according to dichotomized MELD in the whole population and according to amyloid etiology are reported in the ESM Figs. 1 and 2. As visually evident, the two curves split early according to MELD cutoff with a significant divergence in overall study population and in the two etiology cohorts. Moreover, it is notable as the survival falls rapidly both in AL and TTR patients. At the end of the follow-up in the whole study cohort the MELD >12 patients have a significantly lower survival compared to other patients (40.7 vs $66.3 \%$ ), as

Table 2 Differences in clinical, biohumoral and echocardiographic characteristics between dead or alive patients

\begin{tabular}{|c|c|c|c|}
\hline & Alive $(n=78)$ & Dead $(n=50)$ & $p$ \\
\hline MELD & $9.1 \pm 2.7$ & $11.5 \pm 4.6$ & $<0.001$ \\
\hline Age (years) & $71.8 \pm 10.6$ & $69.7 \pm 10.5$ & 0.289 \\
\hline Gender M/F & $50 / 28$ & $22 / 28$ & 0.352 \\
\hline Etiology (AL-TTR) & $45 / 33$ & $37 / 13$ & 0.061 \\
\hline \multicolumn{4}{|l|}{ NYHA class } \\
\hline I & 34 & 9 & \\
\hline II & 28 & 11 & \multirow[t]{3}{*}{$<0.00$} \\
\hline III & 16 & 19 & \\
\hline IV & 0 & 11 & \\
\hline NT-proBNP ng/l & $2625 \pm 3151$ & $9519 \pm 10897$ & $<0.001$ \\
\hline Troponin ng/l & $0.10 \pm 0.12$ & $0.28 \pm 0.42$ & 0.006 \\
\hline Creatinine $\mathrm{mg} / \mathrm{dl}$ & $1.1 \pm 0.4$ & $1.34 \pm 0.5$ & 0.070 \\
\hline LVmass $\mathrm{g} / \mathrm{m}^{2}$ & $154.9 \pm 57.2$ & $165.8 \pm 51.1$ & 0.251 \\
\hline $\mathrm{LA}$ area $\mathrm{cm}^{2}$ & $22.4 \pm 5.6$ & $24.1 \pm 4.5$ & 0.062 \\
\hline $\mathrm{E} / \mathrm{e}^{\prime}$ & $15.2 \pm 6.4$ & $18.2 \pm 8.1$ & 0.002 \\
\hline DT ms & $200 \pm 73$ & $186 \pm 75$ & 0.302 \\
\hline LVEDD mm & $46.7 \pm 5.5$ & $43.6 \pm 5.2$ & 0.002 \\
\hline LVESD mm & $30.3 \pm 6.4$ & $29.9 \pm 5.9$ & 0.637 \\
\hline LVEDV ml & $88.1 \pm 25.4$ & $77.8 \pm 20.5$ & 0.010 \\
\hline LVESV ml & $39.2 \pm 18.1$ & $37.3 .4 \pm 17.6$ & 0.572 \\
\hline $\mathrm{EF} \%$ & $56.4 \pm 9.8$ & $52.6 \pm 12.4$ & 0.041 \\
\hline TAPSE mm & $19.5 \pm 3.9$ & $16.4 \pm 5.2$ & $<0.001$ \\
\hline PASP $\mathrm{mmHg}$ & $33.5 \pm 11.4$ & $39.7 \pm 10.8$ & 0.003 \\
\hline
\end{tabular}

$E$ early diastolic mitral peak flow velocity, $e^{\prime}$ early diastolic peak velocity at lateral mitral annulus, $E F$ ejection fraction, $L A$ left atrium, $L V$ left ventricular, $L V E D D$ LV end-diastolic diameter, $L V E D V$ LV end-diastolic volume, LVESD LV end-systolic diameter, LVESV LV end-systolic volume, PASP pulmonary artery systolic pressure, TAPSE tricuspid annulus systolic plane excursion well as in the two etiology cohorts (AL cohort: 30.8 vs $59.7 \%$; TTR cohort: 50.0 vs $80.6 \%$ ).

The ROC survival results of MELD score, NT-proBNP and troponin for whole population and amyloidosis etiologies are reassessed in Table 3. MELD AUC is inferior to NT-proBNP and troponin AUC in the whole population and in AL patients; conversely in TTR subjects, the MELD score reaches a large AUC compared to other variables with NT-proBNP obtaining the worst result.

\section{Univariate and multivariate analysis}

At univariate analysis, several variables are significantly related to survival, i.e., NYHA class $p<0.0001$; troponin $p<0.0001$, NT-proBNP $<0.001$, LV mass BSA indexed $p<0.028$, TAPSE $p<0.0001$, LV EF $p<0.002$, E/e' $p<0.0001$, MELD as dichotomous variable $p<0.008$ and MELD as continuous variable $p<0.0001$.

In Table 4 we report the results of the two different Cox regression multivariate analysis models in which MELD entered as continuous parameter (model 1) and dichotomous one (model 2), respectively. In both models, the MELD scores have an independent predictive power after adjustment of overall well-known variables able to influence the prognosis in amyloidosis.

\section{Discussion}

This study demonstrates that the MELD scoring system is able to predict prognosis in patients with amyloidosis. Our data show that a MELD score $>12$ selects a high-risk subgroup of amyloidosis patients with poor prognosis during the follow-up with a independent risk of 2.7-fold higher compared to the subgroup with MELD $\leq 12$. Interestingly, for each point of increase of MELD score at baseline, the risk of death rises $19.9 \%$ in the follow-up.

Our finding is in accordance with the results of Kim and colleagues [16] that highlight the prognostic role of MELD in heart failure patients. In fact they demonstrate that a MELD scoring system above 12 is independently associated with $10 \%$ excess of risk for heart transplantation in ambulatory patients with heart failure.

This parallelism may be due to the fact that the onset and the worsening of heart failure are often final cause of death in amyloidosis patients [13]. Furthermore, we know how heart failure is a systemic disease and not simply a cardiovascular disease [28] in which a key prognostic point is related to the onset of cardio-hepatic or cardio-renal syndromes [22, 23]. On this basis, the MELD scoring system is an easy and rapid instrument that can be used in the risk stratification of amyloidosis patients; moreover, the combination of the MELD score and cardiovascular 
Table 3 Receiver operating characteristics (ROC) analysis of MELD, troponin and NT-proBNP in study population and in the two amyloidosis etiologies

\begin{tabular}{|c|c|c|c|c|c|c|}
\hline & \multicolumn{2}{|l|}{ All population } & \multicolumn{2}{|l|}{ AL amyloidosis } & \multicolumn{2}{|l|}{ TTR amyloidosis } \\
\hline & AUC (CI) & $p$ & AUC (CI) & $p$ & AUC (CI) & $p$ \\
\hline MELD & $0.647(0.536-0.758)$ & 0.012 & $0.650(0.497-0.763)$ & 0.041 & $0.853(0.717-0.989)$ & 0.002 \\
\hline NT-proBNP & $0.710(0.604-0.816)$ & $<0.001$ & $0.749(0.628-0.870)$ & $<0.001$ & $0.659(0.449-0.868)$ & 0.658 \\
\hline Troponin & $0.682(0.576-0.788)$ & 0.002 & $0.716(0.592-0.839)$ & 0.002 & $0.726(0.546-0.907)$ & 0.044 \\
\hline
\end{tabular}

Table 4 Independent predictors of survival in the study population using MELD variable as continuous (MODEL 1) and dichotomous (MODEL 2)

\begin{tabular}{|c|c|c|c|c|c|c|}
\hline & \multicolumn{3}{|l|}{ Model 1} & \multicolumn{3}{|l|}{ Model 2} \\
\hline & $\mathrm{B} \pm \mathrm{SE}$ & $p$ & HR (95\%CI) & $\mathrm{B} \pm \mathrm{SE}$ & $p$ & HR (95 \%CI) \\
\hline Gender & $0.63 \pm 0.51$ & 0.217 & $1.437 ;(0.831-2.254)$ & $0.30 \pm 0.25$ & 0.234 & $1.349 ;(0.824-2.208)$ \\
\hline Age & $0.01 \pm 0.02$ & 0.959 & $1.001 ;(0.959-1.045)$ & $-0.01 \pm 0.20$ & 0.830 & $0.996 ;(0.957-1.030)$ \\
\hline NYHA class & $-3.46 \pm 0.98$ & $<0.001$ & $0.040 ;(0.005-0.215)$ & $-2.96 \pm 0.96$ & 0.002 & $0.052 ;(0.008-0.338)$ \\
\hline NT_proBNP & $0.00 \pm 0.00$ & 0.088 & $1.000 ;(1.000-1.000)$ & $0.00 \pm 0.00$ & 0.025 & $1.000 ;(1.000-1.000)$ \\
\hline Troponin & $0.14 \pm 1.04$ & 0.894 & $1.150 ;(0.149-8.889)$ & $-0.09 \pm 1.05$ & 0.930 & $0.913 ;(0.118-7.081)$ \\
\hline LVmass & $-0.01 \pm 0.01$ & 0.471 & 0.995; (0.981-1.009) & $-0.01 \pm 0.01$ & 0.282 & $0.993 ;(0.980-1.006)$ \\
\hline $\mathrm{EF} \%$ & $-0.05 \pm 0.02$ & 0.016 & $0.955 ;(0.919-0.991)$ & $-0.05 \pm 0.02$ & 0.011 & $0.951 ;(0.916-0.988)$ \\
\hline $\mathrm{E} / \mathrm{e}^{\prime}$ & $0.01 \pm 0.04$ & 0.892 & $1.005 ;(0.938-1.076)$ & $0.00 \pm 0.03$ & 0.981 & $1.001 ;(0.938-1.068)$ \\
\hline TAPSE mm & $0.04 \pm 0.07$ & 0.549 & $1.040 ;(0.915-1.181)$ & $0.02 \pm 0.06$ & 0.813 & $1.015 ;(0.899-1.145)$ \\
\hline AL vs TTR & $-0.81 \pm 0.79$ & 0.303 & $0.444 ;(0.095-2.078)$ & $-0.52 \pm 0.72$ & 0.471 & $0.594 ;(0.144-2.449)$ \\
\hline MELD $^{\mathrm{a}}$ & $0.18 \pm 0.05$ & 0.001 & $1.199 ;(1.082-1.329)$ & & & \\
\hline MELD > 12 & & & & $1.00 \pm 0.47$ & 0.035 & $2.707 ;(1.075-6.817)$ \\
\hline
\end{tabular}

$E$ early diastolic mitral peak flow velocity, $e^{\prime}$ early diastolic peak velocity at lateral mitral annulus, $E F$ ejection fraction, $M E L D$ model for endstage liver disease, TAPSE tricuspid annulus systolic plane excursion

${ }^{\text {a }}$ Considered as continuous variable

variables, exploring different clinical domains, might give significant additive prognostic information in these patients.

Another important result is related to the finding that for each point of increase of MELD, the patient risk of death rises significantly; this result may open an interesting perspective for MELD scoring system in the managing of amyloidosis patients. In fact as demonstrated in cirrhotic patients, the MELD scoring system might be used to continuously evaluate the amyloidosis patients in the followup visits with the aim of capturing an early significant modification of the liver and renal global functions with the same prognostic perspective that we often attribute to the self-reported cardiovascular functional evaluation described with the NYHA classification.

Furthermore, as described by ROC curve analysis, the MELD scores, although always statistically significant, show a lower sensitivity/specificity ratio than those of troponin I and NT-proBNP in the whole study population and AL subgroup. Conversely, in TTR subgroup of patients, the MELD scores seem to have the larger AUC compared to Troponin and NT-proBNP. It is notable that the MELD scores reach the best accuracy in survival prediction in a TTR patient in whom currently no effective scoring systems have been validated.

These best results in the TTR cohort could be due to the fact that no clinical significant liver or kidney infiltration is detectable in TTR amyloidosis, therefore it could be hypothesized that in a TTR patient, the MELD score captures the worsening of liver and kidney function specifically due to the heart failure pathophysiological cascade related to cardio-renal or cardio-hepatic syndrome.

In AL amyloidosis, a MELD score increase, related mainly to rise in creatinine value could reflect a parenchymal kidney injury, and not be a marker of cardiorenal syndrome or glomerular venous congestion [23]. In fact no significant correlation is observed in AL patients between MELD scores and right ventricular function or pulmonary pressure (TAPSE and PASP, respectively, Table 5 in Appendix data). Conversely in TTR patients, we 
find a strong bivariate correlation between MELD and TAPSE/PASP (Additional supplement Table).

\section{Limitation}

This paper reflects the limitations pertaining to retrospective analysis, therefore MELD scores need to be validated in a longitudinal prospective study prior to being considered for clinical practice.

Our choice to put together ATTRm and ATTRwt patients might be criticized. However, in our population the TTRm and TTRwt phenotypes were similar in cardiac involvement, echocardiographic profile and age at diagnosis [20, 29-31]. A further limitation is lack of biopsy evidence of amyloid in $32 \%$ of TTRwt population. However, this cohort of patients was fully characterized with all other clinical investigative techniques currently available. When combined these are known to provide high diagnostic accuracy [32, 33].

\section{Conclusion}

Our study demonstrated that MELD scoring system is able to predict prognosis in patients with amyloidosis. Our data show that MELD has a lower sensitivity/specificity ratio in survival prediction than those of troponin I and NT-proBNP in whole study population and AL subgroup. On the other hand, it is notable that MELD reaches the best accuracy in survival prediction in TTR patients, a subgroup in whom currently no effective scoring systems have been validated.

\section{Compliance with ethical standards}

\section{Conflict of interest None.}

Statement of human and animal rights For this type of study formal consent is not required.

Informed consent All patients gave written informed consent for their clinical records to be used for research purposes, in accordance with Institutional Review Board guidelines.

\section{Appendix}

\section{See Table 5 .}

Table 5 Relationship between MELD score (used as continuous variable), and right ventricular variables according to amyloidosis etiologies

\begin{tabular}{cccccc}
\hline & AL cohort & & & TTR cohort \\
\cline { 2 - 3 } \cline { 5 - 5 } \cline { 5 - 6 } TAPSE & PAPS & & TAPSE & PASP \\
\hline MELD & $\begin{array}{c}R:-0.17 ; \\
p=0.150\end{array}$ & $\begin{array}{c}R: 0.18 ; \\
p=0.130\end{array}$ & $\begin{array}{c}R:-0.55 ; \\
p<0.001\end{array}$ & $\begin{array}{c}R: 0.40 ; \\
p=0.010\end{array}$ \\
\hline
\end{tabular}

\section{References}

1. Kumar S, Dispenzieri A, Lacy MQ, Hayman SR, Buadi FK, Colby C, Laumann K, Zeldenrust SR, Leung N, Dingli D, Greipp PR, Lust JA, Russell SJ, Kyle RA, Rajkumar SV, Gertz MA (2012) Revised prognostic staging system for light chain amyloidosis incorporating cardiac biomarkers and serum free light chain measurements. J Clin Oncol 30:989-995

2. Dispenzieri A, Gertz MA, Kyle RA, Lacy MQ, Burritt MF, Therneau TM, Greipp PR, Witzig TE, Lust JA, Rajkumar SV, Fonseca R, Zeldenrust SR, McGregor CG, Jaffe AS (2004) Serum cardiac troponins and $\mathrm{N}$-terminal pro-brain natriuretic peptide: a staging system for primary systemic amyloidosis. J Clin Oncol 22:3751-3757

3. Kumar SK, Gertz MA, Lacy MQ, Dingli D, Hayman SR, Buadi FK, Short-Detweiler K, Zeldenrust SR, Leung N, Greipp PR, Lust JA, Russell SJ, Kyle RA, Rajkumar SV, Dispenzieri A (2011) Recent improvements in survival in primary systemic amyloidosis and the importance of an early mortality risk score. Mayo Clin Proc 86:12-18

4. Kristen AV, Scherer K, Buss S, Aus dem Siepen F, Haufe S, Bauer R, Hinderhofer K, Giannitsis E, Hardt S, Haberkorn U, Katus HA, Steen H (2014) Noninvasive risk stratification of patients with transthyretin amyloidosis. JACC Cardiovasc Imaging 7:502-510

5. Palladini G, Lavatelli F, Russo P, Russo P, Perlini S, Perfetti V, Bosoni T, Obici L, Bradwell AR, D'Eril GM, Fogari R, Moratti R, Merlini G (2006) Circulating amyloidogenic free light chains and serum $\mathrm{N}$-terminal natriuretic peptide type $\mathrm{B}$ decrease simultaneously in association with improvement of survival in AL. Blood 107:3854-3858

6. Palladini G, Barassi A, Klersy C, Pacciolla R, Milani P, Sarais G, Perlini S, Albertini R, Russo P, Foli A, Bragotti LZ, Obici L, Moratti R (2010) Melzi d'Eril GV, Merlini G. The combination of high-sensitivity cardiac troponin $\mathrm{T}$ (hs-cTnT) at presentation and changes in N-terminal natriuretic peptide type B (NTproBNP) after chemotherapy best predicts survival in AL amyloidosis. Blood 116:3426-3430

7. Dispenzieri A, Gertz MA, Kumar SK, Lacy MQ, Kyle RA, Saenger AK, Grogan M, Zeldenrust SR, Hayman SR, Buadi F, Greipp PR, Leung N, Russell SR, Dingli D, Lust JA, Rajkumar SV, Jaffe AS (2014) High sensitivity cardiac troponin $T$ in patients with immunoglobulin light chain amyloidosis. Heart 100:383-388

8. Buss SJ, Emami M, Mereles D, Korosoglou G, Kristen AV, Voss A, Schellberg D, Zugck C, Galuschky C, Giannitsis E, Hegenbart U, Ho AD, Katus HA, Schonland SO, Hardt SE (2012) Longitudinal left ventricular function for prediction of survival in systemic light-chain amyloidosis: incremental value compared with clinical and biochemical markers. J Am Coll Cardiol 60:1067-1076

9. Bellavia D, Pellikka PA, Dispenzieri A, Scott CG, Al-Zahrani GB, Grogan M, Pitrolo F, Oh JK, Miller FA Jr (2012) Comparison of right ventricular longitudinal strain imaging, tricuspid annular plane systolic excursion, and cardiac biomarkers for early diagnosis of cardiac involvement and risk stratification in primary systematic (AL) amyloidosis: a 5-year cohort study. Eur Heart J Cardiovasc Imaging 13:680-689

10. Bellavia D, Abraham RS, Pellikka PA, Dispenzieri A, Burnett JC Jr, Al-Zahrani GB, Green TD, Manske MK, Gertz MA, Miller FA Jr, Abraham TP (2011) Utility of Doppler myocardial imaging, cardiac biomarkers, and clonal immunoglobulin genes to assess left ventricular performance and stratify risk following peripheral blood stem cell transplantation in patients with systemic light chain amyloidosis (Al). J Am Soc Echocardiogr 24:444-454 
11. Perlini S, Salinaro F, Musca F, Mussinelli R, Boldrini M, Raimondi A, Milani P, Foli A, Cappelli F, Perfetto F, Palladini G, Rapezzi C, Merlini G (2014) Prognostic value of depressed midwall systolic function in cardiac light-chain amyloidosis. J Hypertens 32:1121-1131

12. Cappelli $\mathrm{F}$, Porciani MC, Bergesio $\mathrm{F}$, Perlini $\mathrm{S}$, Attanà $\mathrm{P}$, Moggi Pignone A, Salinaro F, Musca F, Padeletti L, Perfetto F (2012) Right ventricular function in AL amyloidosis: characteristics and prognostic implication. Eur Heart $\mathbf{J}$ Cardiovasc Imaging $13: 416-422$

13. Perfetto F, Cappelli F, Bergesio F, Ciuti G, Porciani MC, Padeletti L, Moggi Pignone A (2013) Cardiac amyloidosis: the heart of the matter. Intern Emerg Med 8:191-203

14. Kamath PS, Wiesner RH, Malinhoc M, Kremers W, Therneau TM, Kosberg CL, D'Amico G, Dickson ER, Kim WR (2001) A model to predict survival in patients with end-stage liver disease. Hepatology 33:464-470

15. Wiesner R, Edwards E, Freeman R, Harper A, Kim R, Kamath P, Kremers W, Lake J, Howard T, Merion RM, Wolfe RA, Krom R, United Network for Organ Sharing Liver Disease Severity Score Committee (2003) Model for end-stage liver disease (MELD) and allocation of donor livers. Gastroenterology 124:91-96

16. Kim MS, Kato TS, Farr M, Wu C, Givens RC, Collado E, Mancini DM, Schulze PC (2013) Hepatic dysfunction in ambulatory patients with heart failure: application of the MELD scoring system for outcome prediction. J Am Coll Cardiol 61:2253-2261

17. Bonde $\mathrm{P}, \mathrm{Ku} \mathrm{NC}$, Genovese EA, Bermudez CA, Bhama JK, Ciarleglio MM, Cong X, Teuteberg JJ, Kormos RL (2012) Model for end-stage liver disease score predicts adverse events related to ventricular assist device therapy. Ann Thorac Surg 93:1541-1547

18. Yang JA, Kato TS, Shulman BP, Takayama H, Farr M, Jorde UP, Mancini DM, Naka Y, Schulze PC (2012) Liver dysfunction as a predictor of outcomes in patients with advanced heart failure requiring ventricular assist device support: use of the Model of End-stage Liver Disease (MELD) and MELD eXcluding INR (MELD-XI) scoring system. J Heart Lung Transplant 31:601-610

19. Tsuda K, Koide M, Kunii Y, Watanabe K, Miyairi S, Ohashi Y, Harada T (2013) Simplified model for end-stage liver disease score predicts mortality for tricuspid valve surgery. Interact CardioVasc Thorac Surg 16:630-635

20. Kato TS, Stevens GR, Jiang J, Schulze PC, Gukasyan N, Lippel M, Levin A, Homma S, Mancini D, Farr M (2013) Risk stratification of ambulatory patients with advanced heart failure undergoing evaluation for heart transplantation. J Heart Lung Transplant 32:333-340

21. Chokshi A, Cheema FH, Schaefle KJ, Jiang J, Collado E, Shahzad K, Khawaja T, Farr M, Takayama H, Naka Y, Mancini DM, Schulze PC (2012) Hepatic dysfunction and survival after orthotopic heart transplantation: application of the MELD scoring system for outcome prediction. J Heart Lung Transplant 31:591-600

22. Van Deursen VM, Damman K, Hillege HL, van Beek AP, van Veldhuisen DJ, Voors AA (2010) Abnormal liver function in relation to hemodynamic profile in heart failure patients. J Card Fail 16:84-90

23. Ronco C, Cicoira M, McCullough PA (2012) Cardiorenal syndrome type 1: pathophysiological crosstalk leading to combined heart and kidney dysfunction in the setting of acutely decompensated heart failure. J Am Coll Cardiol 60:1031-1042

24. Cappelli F, Baldasseroni S, Bergesio F, Padeletti L, Attanà $\mathrm{P}$, Pignone AM, Grifoni E, Ciuti G, Fabbri A, Tarantini F, Marchionni N, Gensini GF, Perfetto F (2014) Biohumoral markers as predictor of right ventricular dysfunction in AL Amyloidosis. Amyloid 21:97-102

25. Falk RH (2005) Diagnosis and management of the cardiac amyloidoses. Circulation 112:2047-2060

26. Perugini E, Guidalotti PL, Salvi F, Cooke RM, Pettinato C, Riva L, Leone O, Farsad M, Ciliberti P, Bacchi-Reggiani L, Fallani F, Branzi A, Rapezzi C (2005) Non invasive etiologic diagnosis of cardiac amyloidosis using 99mTc-3,3-diphosphono-1,2-propanodicarboxylic acid scintigraphy. J Am Coll Cardiol 46:10761084

27. Lang RM, Bierig M, Devereux RB, Flachskampf FA, Foster E, Pellikka PA, Picard MH, Roman MJ, Seward J, Shanewise JS, Solomon SD, Spencer KT, Sutton MS, Stewart WJ, Chamber Quantification Writing Group; American Society of Echocardiography's Guidelines and Standards Committee; European Association of Echocardiography (2005) Recommendations for chamber quantification: a report from the American Society of Echocardiography's Guidelines and Standards Committee and the Chamber Quantification Writing Group, developed in conjunction with the European Association of Echocardiography, a branch of the European Society of Cardiology. J Am Soc Echocardiogr 18:1440-1463

28. Braunwald E (2013) Heart Failure. JACC Heart Fail 1:1-20

29. Cappelli F, Baldasseroni S, Bergesio F, Perlini S, Salinaro F, Padeletti L, Attanà $\mathrm{P}$, Paoletti Perini A, Moggi Pignone A, Grifoni E, Fabbri A, Marchionni N, Gensini GF, Perfetto F (2015) Echocardiographic and biohumoral characteristics in patients with AL and TTR amyloidosis at diagnosis. Clin Cardiol. 38(2):69-75

30. Cappelli F, Frusconi S, Bergesio F, Grifoni E, Fabbri A, Giuliani C, Falconi S, Bonifacio S, Perfetto F (2016) The Val142Ile transthyretin cardiac amyloidosis: not only an Afro-American pathogenic variant? A single-centre Italian experience. J Cardiovasc Med (Hagerstown). 17(2):122-125

31. Perfetto F, Cappelli F, Bergesio F (2011) Asymptomatic homozygous gene carrier in a family with Ile68Leu ATTR amyloidosis: a new endemic region in northern Tuscany? J Cardiovasc Med (Hagerstown) 12(6):450-451

32. Fontana M, Banypersad SM, Treibel TA, Maestrini V, Sado DM, White SK, Pica S et al (2014) Native T1 mapping in transthyretin amyloidosis. JACC Cardiovasc Imaging 7:157-165

33. Hutt DF, Quigley AM, Page J, Hall ML, Burniston M, Gopaul D, Lane $\mathrm{T}$ et al (2014) Utility and limitations of 3,3-diphosphono1,2-propanodicarboxylic acid scintigraphy in systemic amyloidosis. Eur Heart J Cardiovasc Imaging 15:1289-1298 